\title{
An FPGA-Integrated Time-to-Digital Converter Based on a Ring Oscillator for Programmable Delay Line Resolution Measurement
}

\author{
Chao Chen, ${ }^{1,2}$ Shengwei Meng, ${ }^{1}$ Zhenghuan Xia, ${ }^{1,2}$ Guangyou Fang, ${ }^{1}$ and Hejun Yin ${ }^{3}$ \\ ${ }^{1}$ Key Laboratory of Electromagnetic Radiation and Sensing Technology, Chinese Academy of Sciences, No. 19, \\ North 4th Ring Road West, Haidian District, Beijing 100190, China \\ ${ }^{2}$ University of Chinese Academy of Sciences, No. 19A, Yuquan Road, Beijing 100049, China \\ ${ }^{3}$ Chinese Academy of Sciences, 52 Sanlihe Road, Beijing 100864, China \\ Correspondence should be addressed to Chao Chen; iecaschenchao@sina.com.cn
}

Received 25 February 2014; Revised 21 March 2014; Accepted 26 March 2014; Published 13 April 2014

Academic Editor: J. S. Mandeep

Copyright (C) 2014 Chao Chen et al. This is an open access article distributed under the Creative Commons Attribution License, which permits unrestricted use, distribution, and reproduction in any medium, provided the original work is properly cited.

\begin{abstract}
We describe the architecture of a time-to-digital converter (TDC), specially intended to measure the delay resolution of a programmable delay line (PDL). The configuration, which consists of a ring oscillator, a frequency divider (FD), and a period measurement circuit (PMC), is implemented in a field programmable gate array (FPGA) device. The ring oscillator realized in loop containing a PDL and a look-up table (LUT) generates periodic oscillatory pulses. The FD amplifies the oscillatory period from nanosecond range to microsecond range. The time-to-digital conversion is based on counting the number of clock cycles between two consecutive pulses of the FD by the PMC. Experiments have been conducted to verify the performance of the TDC. The achieved relative errors for four PDLs are within $0.50 \%-1.21 \%$ and the TDC has an equivalent resolution of about 0.4 ps.
\end{abstract}

\section{Introduction}

Many radar systems such as pulsed radar [1], ultra-wideband (UWB) radar [2, 3], random noise radar [4], and multiple input multiple output (MIMO) radar [5] require timing generator circuits to make timing adjustment. Programmable delay line (PDL) is often being applied as a high precision timing circuitry to introduce both coarse and fine delays. The performance of these radar systems depends a lot on the delay resolution of the PDL. However, the actual delay resolution of a PDL may differ slightly from its nominal delay resolution due to changes in temperature, power voltage, and other factors. To ensure correct timing, high resolution measurement of a PDL is essential before design begins. Since there is no direct way to realize the measurement of the delay resolution, it can be converted into time interval measurement by injecting two synchronized rising edges into two PDLs with different delays and connecting the two outputs of two PDLs to an XOR gate. Thus, the XOR gate yields a pulse signal with a pulse width related to the delay resolution. Then we can measure the pulse width to indirectly get the resolution. Time-to-digital converter (TDC) is being widely used for time interval measurement and TDC can be easily implemented by a digital device, such as a field programmable gate array (FPGA) or an application-specific integrated circuit (ASIC). Most available TDCs with picosecond resolution are implemented in ASIC technology as the technology allows precise control of the internal propagation times for signal. Seo et al. introduced a cyclic TDC with 1.25 ps resolution [6], whereas Kim et al. presented a pipelined TDC with 1.12 ps resolution [7]. Xu et al. described a TDC providing $0.84 \mathrm{ps}$ resolution [8]. However, these TDCs have a very high cost of development and are not easily adaptable to new applications. In comparison with ASIC-based TDCs, FPGA-integrated designs [9-12] lack competitiveness in terms of resolution, although they are easily adaptable to a new application and have shorter development cycle and lower development cost. Therefore, an FPGA-integrated TDC with picosecond resolution is of great importance. This paper describes a TDC implemented in a 


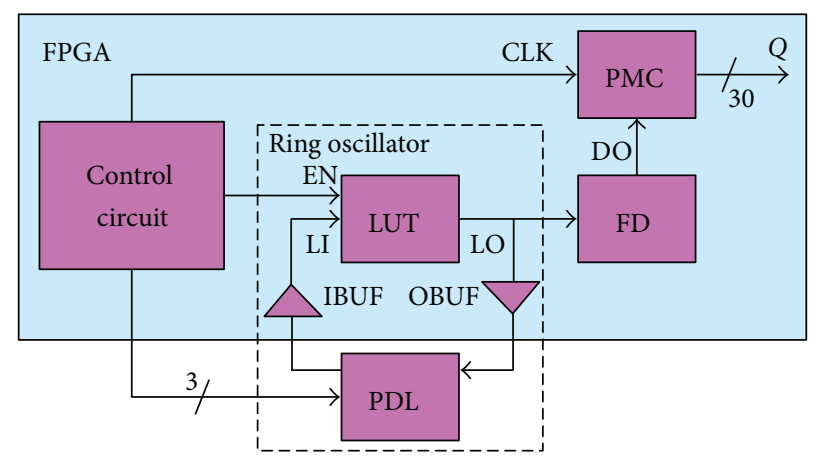

FIGURE 1: Block diagram of the proposed FPGA-integrated TDC.

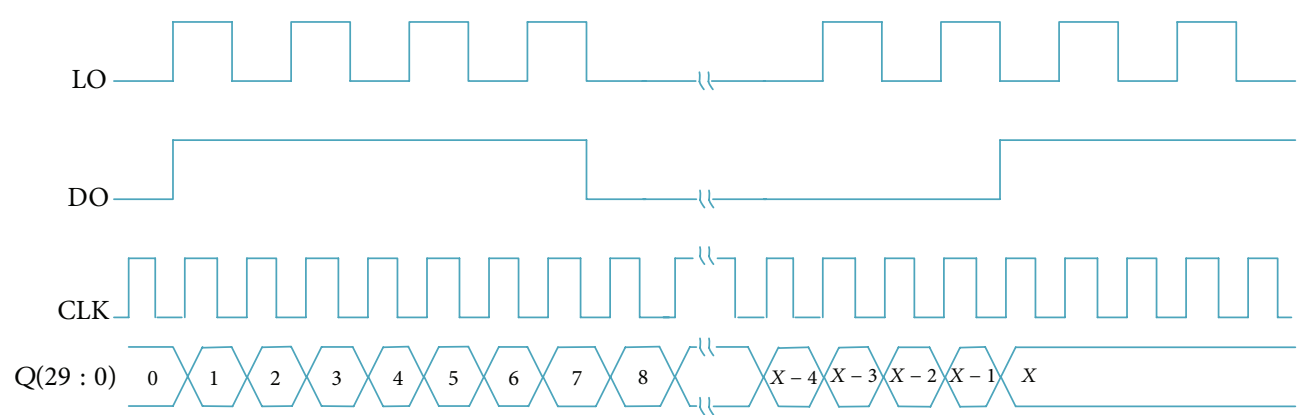

FIgURE 2: Timing diagram of the proposed FPGA-integrated TDC.

TABLE 1: Logic table of LUT.

\begin{tabular}{lcc}
\hline Input & & Output \\
EN & LI & LO \\
\hline 0 & 0 & 1 \\
0 & 1 & 1 \\
1 & 0 & 1 \\
1 & 1 & 0 \\
\hline
\end{tabular}

Xilinx FPGA device to measure the actual delay resolution of a PDL and it achieves an equivalent resolution of about 0.4 ps.

\section{Operation Principles}

A block diagram of the proposed FPGA-integrated TDC is illustrated in Figure 1. It is composed of three blocks, namely, the ring oscillator, the frequency divider (FD), and the period measurement circuit (PMC). The ring oscillator consists of a PDL, an input buffer (IBUF), an output buffer (OBUF), and a look-up table (LUT) with two inputs and one output. The logic table of the LUT is presented in Table 1.

The signal with a period of nanosecond range from the output of the ring oscillator needs to be amplified to microsecond range by the FD before it enters into the PMC. The FD has a divide ratio of $D$ and the PMC involves the use of a counter driven by a reference clock CLK of period $T_{\mathrm{CLK}}$. The time-to-digital conversion is based on counting the number of clock cycles between two consecutive pulses of the FD by the PMC. Timing diagram of the FPGA-integrated TDC is shown in Figure 2.

The oscillation period of the ring oscillator derived using the most common way is given as follows:

$$
T=2\left(t_{0}+t_{1}+t_{2}\right)
$$

where $t_{0}$ is the variable time delay PDL yields, $t_{1}$ is the constant time delay PDL yields, and $t_{2}$ is the time required by the signal to propagate from the output of the PDL to the input of the PDL. Delays $t_{1}$ and $t_{2}$ are constants, while $t_{0}$ is programmable to be modified. The period of the FD's output signal is given by

$$
T_{0}=D \times T
$$

The PMC measures the period $T_{0}$ to obtain the result $X=$ $T_{0} / T_{\text {CLK }}$, where $X$ is an unsigned decimal integer at the PMC output.

In the proposed FPGA-integrated TDC, when the variable time delay of the PDL is modified, the outputs of the three blocks would change. When the variable time delay of the PDL is programmed to $t_{01}$, the signal period of the ring oscillator is recalculated using

$$
T_{1}=2\left(t_{01}+t_{1}+t_{2}\right)
$$

where $t_{01}=Y_{1} \times \Delta T, Y_{1}$ is a control word generated by FPGA and $\Delta T$ is the actual delay resolution of the PDL. The PMC output can be written as $X_{1}=D \times T_{1} / T_{\text {CLK }}$. 


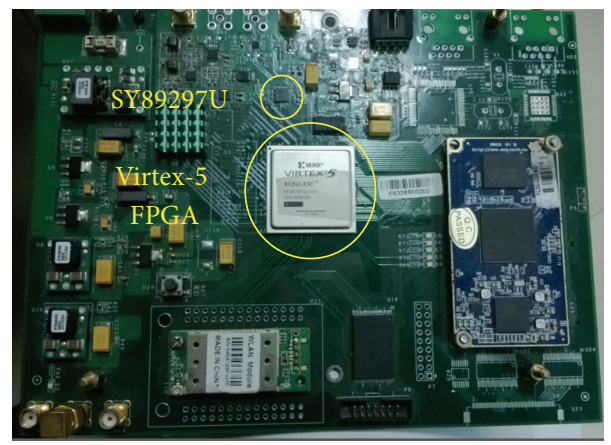

FIgURE 3: Photograph of the radar board.

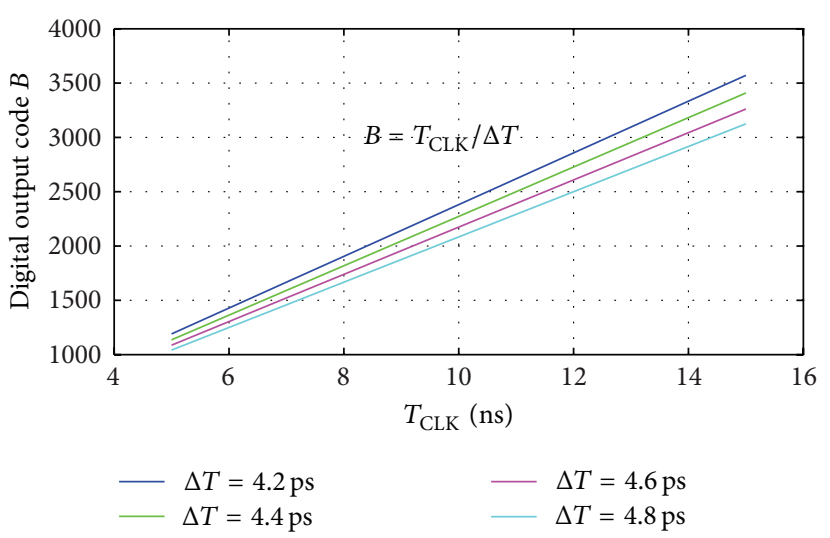

(a) Different $\Delta T$

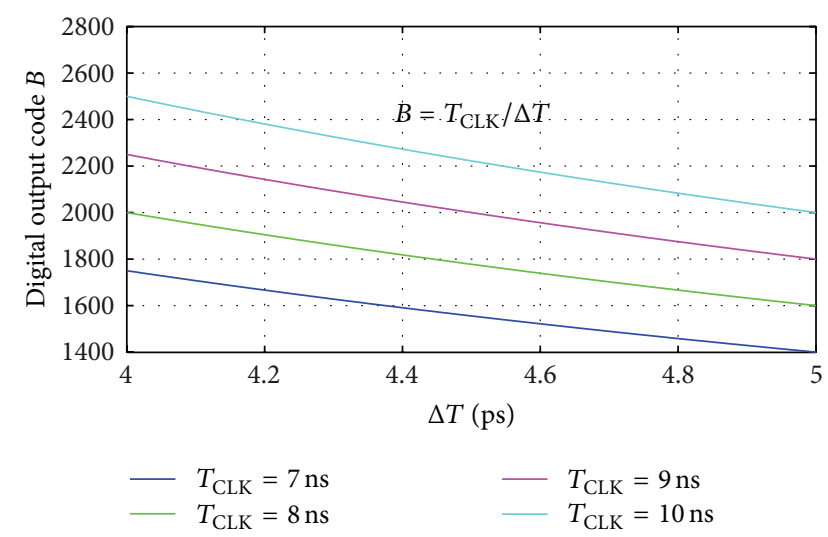

(b) Different $T_{\text {CLK }}$

FIgURE 4: Simulated characteristic curves of the proposed TDC.

When the variable time delay of the PDL is programmed to $t_{02}$, the signal period of the ring oscillator is then recalculated using

$$
T_{2}=2\left(t_{02}+t_{1}+t_{2}\right)
$$

where $t_{02}=Y_{2} \times \Delta T$ and $Y_{2}$ is a control word generated by FPGA. The PMC output can be written as $X_{2}=D \times T_{2} / T_{\text {CLK }}$.

By combining (3) and (4), the actual delay resolution $\Delta T$ can be calculated using

$$
\begin{aligned}
\Delta T & =\frac{\left(T_{1}-T_{2}\right)}{2\left(Y_{1}-Y_{2}\right)} \\
& =\frac{\left(X_{1}-X_{2}\right) \times T_{\mathrm{CLK}}}{\left(2\left(Y_{1}-Y_{2}\right) \times D\right)} \\
& =\frac{T_{\mathrm{CLK}}}{B},
\end{aligned}
$$

where $B=2\left(Y_{1}-Y_{2}\right) \times D /\left(X_{1}-X_{2}\right)$.

By combining (1) and (2), we can get

$$
\left(t_{0}+t_{1}+t_{2}\right)=\frac{X \cdot T_{\mathrm{CLK}}}{2 D}
$$

Thus, we give an equivalent $\mathrm{TDC}$ resolution of $T_{\mathrm{CLK}} / 2 \mathrm{D}$, since the TDC is intended to measure the delay resolution instead of the time interval.
TABLE 2: Brief comparison of the four PDLs.

\begin{tabular}{lcccc}
\hline PDL number & 1 & 2 & 3 & 4 \\
\hline System clock period $T_{\text {CLK }}(\mathrm{ns})$ & 7.532 & 7.940 & 7.890 & 7.782 \\
Clock uncertainty $\Delta T_{\text {CLK }}$ (ns) & 0.087 & 0.035 & 0.035 & 0.087 \\
Digital output code $B$ & 1650 & 1773 & 1786 & 1726 \\
Absolute error $\Delta B$ & 1 & 1 & 1 & 1 \\
Relative error $(\%)$ & 1.21 & 0.50 & 0.50 & 1.18 \\
Actual delay resolution $\Delta t(\mathrm{ps})$ & 4.56 & 4.48 & 4.42 & 4.51 \\
Nominal delay resolution $\Delta T(\mathrm{ps})$ & 5 & 5 & 5 & 5 \\
\hline
\end{tabular}

\section{Experimental Results}

To evaluate the performance of the proposed converter, we implemented its structure on a radar board equipped with a Virtex-5 FPGA device (Xilinx) and a programmable, twochannel delay line SY89297U (Micrel) as shown in Figure 3. Simulated characteristic curves of the proposed TDC are illustrated in Figure 4. Experiments were performed on four different radar boards at an ambient temperature of about $22^{\circ} \mathrm{C}$ with the use of Xilinx Integrated Software Environment (ISE) 13.2 written in Verilog. We set $Y_{1}=1000, Y_{2}=500$, $D=10000$. The digital output code $B$ was collected using Xilinx ChipScope Pro Analyzer which was also used to verify 
TABLE 3: Brief comparison with previous works.

\begin{tabular}{lccccc}
\hline & {$[6]$} & {$[7]$} & {$[8]$} & \multicolumn{1}{c}{ This work } \\
\hline Structure & Cyclic & True pipeline & SAR-ADC & Vernier & Ring oscillator \\
Process $(\mathrm{nm})$ & 130 & 65 & 65 & 65 & 65 \\
Device & ASIC & ASIC & FPGA & FPGA \\
DNL (LSB) & \pm 0.7 & 1.7 & $-0.7 / 1.0$ & N/A & N/A \\
INL (LSB) & $-3 \sim+1$ & 1.12 & $-2.7 / 1.7$ & $-0.93 \sim 0.75$ & N/A \\
Resolution (ps) & 1.25 & & 0.84 & 1.58 & Equivalent to 0.4 \\
\hline
\end{tabular}

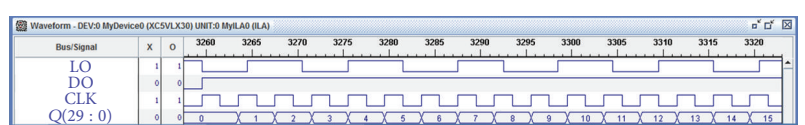

FIGURE 5: Measured timing diagram of the proposed TDC.

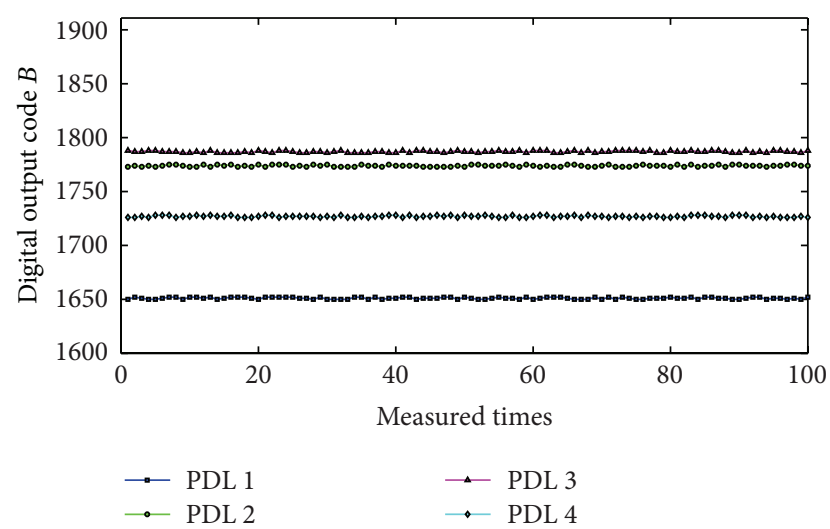

Figure 6: Measurement results of the proposed converter for four PDLs.

the actual timing of the TDC as depicted in Figure 5. Measurements were repeated 100 times during each experiment. The measurement results of the proposed converter for four PDLs are shown in Figure 6. Furthermore, a relative error can be determined. Assume that the clock uncertainty of the system clock is $\Delta T_{\mathrm{CLK}}$ and that the absolute error of the digital output code is $\Delta B$; then the relative error can be calculated as $\left(\Delta T_{\mathrm{CLK}} / T_{\mathrm{CLK}}+\Delta B / B\right)$. Figure 7 shows that the relative errors were measured to be within $0.50 \%-1.21 \%$, which were in a reasonable range for programmable delay line resolution measurement.

Table 2 gives a brief comparison of the four PDLs. As the four PDLs are not measured by the same TDC but separately measured by its corresponding TDC, the reference clocks of the TDCs would demonstrate difference in clock period and clock uncertainty. Furthermore, the FPGAs where the TDCs are implemented also have influence on clock period and clock uncertainty. Thus, the four PDLs show different relative errors. As the system clock period $T_{\mathrm{CLK}}$ is about $8 \mathrm{~ns}$ and the divide ratio $D$ equals 10000 , then the equivalent TDC resolution $T_{\mathrm{CLK}} / 2 \mathrm{D}$ equals about $0.4 \mathrm{ps}$.

Table 3 summarizes the performance comparison of several TDCs. Compared with $[6-8,12]$, the proposed TDC achieves the highest resolution. Hence, this TDC is more

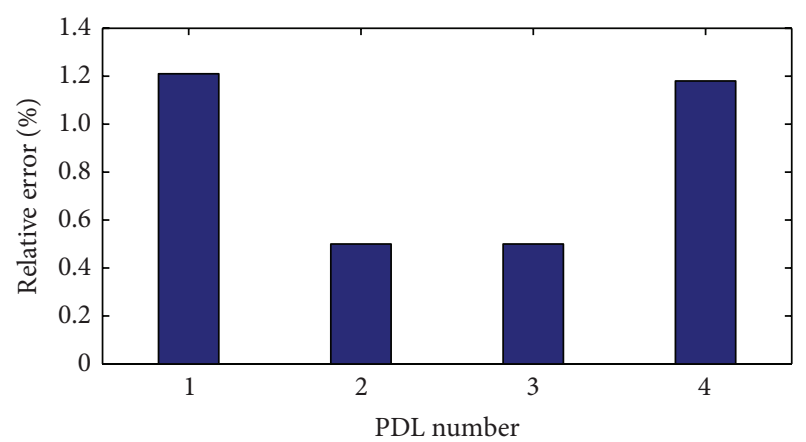

FIgURE 7: Relative error variation for four PDLs.

competitive than other TDCs for programmable delay line resolution measurement.

\section{Conclusion}

We realized an FPGA-integrated TDC with 0.4 ps equivalent resolution using an architecture based on a ring oscillator. It measures the delay resolution of the PDL used in radar systems to ensure correct timing generation. It also provides great operating flexibility as FPGA can allow for an easy configuration to support both delay resolution measurement and radar control. The TDC that we proposed is very promising and well suited for use in radar applications.

\section{Conflict of Interests}

The authors declare that there is no conflict of interests regarding the publication of this paper.

\section{Acknowledgment}

This work was supported by the National High Technology Research and Development Program of China (863 Program: 2012AA121901).

\section{References}

[1] N. Chrétien, J. M. Friedt, G. Martin, and S. Ballandras, "A stroboscopic approach to surface acoustic wave delay line interrogation," in Proceedings of the European Frequency and Time Forum \& International Frequency Control Symposium (EFTF/IFC '13), pp. 771-774, Prague, Czech Republic, July 2013. 
[2] B. Lopes, N. Paulino, J. Goes, and A. Steiger-Garção, "Digitally programmable delay-locked-loop with variable charge pump current," in Proceedings of the 17th International Conference on Mixed Design of Integrated Circuits and Systems (MIXDES '10), pp. 259-264, Warsaw, Poland, June 2010.

[3] N. Paulina, M. Serrazina, J. Goes, and A. Steiger-Garção, "Design of a digitally programmable delay-locked-loop for a low-cost ultra wide band radar receiver," in Proceedings of the IEEE International Symposium on Circuits and Systems, pp. I133-I136, May 2003.

[4] R. M. Narayanan, W. Zhou, K. H. Wagner, and S. Kim, "Acoustooptic correlation processing in random noise radar," IEEE Geoscience and Remote Sensing Letters, vol. 1, no. 3, pp. 166-170, 2004.

[5] M. Z. Dooghabadi, H. A. Hjortland, and T. S. B. Lande, "A linear IR-UWB MIMO radar array," in Proceedings of the IEEE International Conference on Ultra-Wideband (ICUWB '13), pp. 13-19, Sydney, Australia, September 2013.

[6] Y.-H. Seo, J.-S. Kim, H.-J. Park, and J.-Y. Sim, "A 1.25 ps resolution $8 \mathrm{~b}$ cyclic TDC in $0.13 \mu \mathrm{m}$ CMOS," IEEE Journal of Solid-State Circuits, vol. 47, no. 3, pp. 736-743, 2012.

[7] K. S. Kim, W. S. Yu, and S. H. Cho, "A 9b, 1. 12 ps resolution $2.5 \mathrm{~b} /$ stage pipelined time-to-digital converter in $65 \mathrm{~nm}$ CMOS using time-register," in Proceedings of the Symposium on VLSI Circuits (VLSIC '13), pp. C136-C137, Kyoto, Japan, June 2013.

[8] Z. Xu, S. Lee, M. Miyahara, and A. Matsuzawa, "A 0. 84 psLSB $2.47 \mathrm{~mW}$ time-to-digital converter using charge pump and SAR-ADC," in Proceedings of the Custom Integrated Circuits Conference (CICC '13), pp. 1-4, San Jose, Calif, USA, September 2013.

[9] M. P. Mattada and H. Guhilot, "Area efficient vernier Time to Digital Converter (TDC) with improved resolution using identical ring oscillators on FPGA," in Proceedings of the International Conference on Smart Structures and Systems (ICSSS '13), pp. 125-130, Chennai, India, March 2013.

[10] K. J. Hong, E. Kim, J. Y. Yeom, P. D. Olcott, and C. S. Levin, "FPGA-based time-to-digital converter for time-of-flight PET detector," in Proceedings of the Nuclear Science Symposium and Medical Imaging Conference (NSS/MIC '12), pp. 2463-2465, Anaheim, Calif, USA, November 2012.

[11] S. S. Junnarkar, P. O'Connor, P. Vaska, and R. Fontaine, "FPGAbased self-calibrating time-to-digital converter for time-offlight experiments," IEEE Transactions on Nuclear Science, vol. 56, no. 4, pp. 2374-2379, 2009.

[12] P. Chen, P.-Y. Chen, J.-S. Lai, and Y.-J. Chen, "FPGA vernier digital-to-time converter with 1.58 ps resolution and 59.3 minutes operation range," IEEE Transactions on Circuits and Systems I: Regular Papers, vol. 57, no. 6, pp. 1134-1142, 2010. 

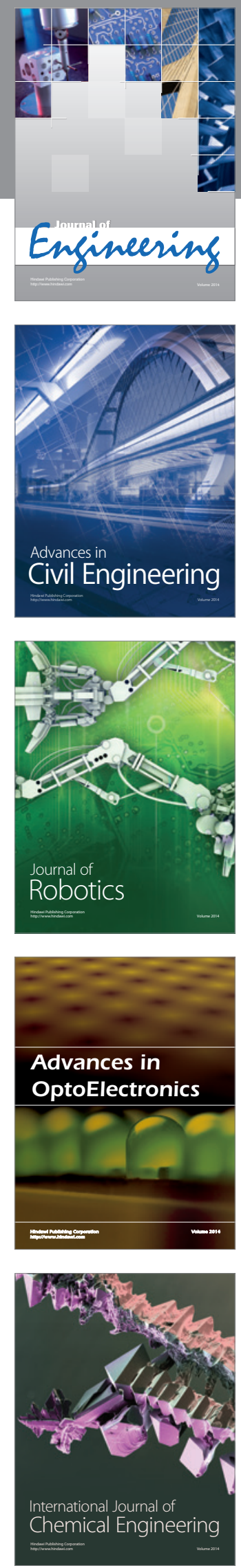

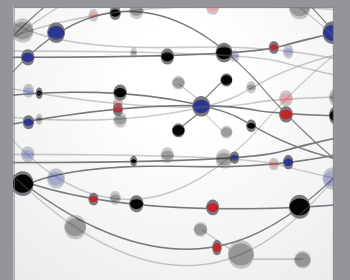

The Scientific World Journal
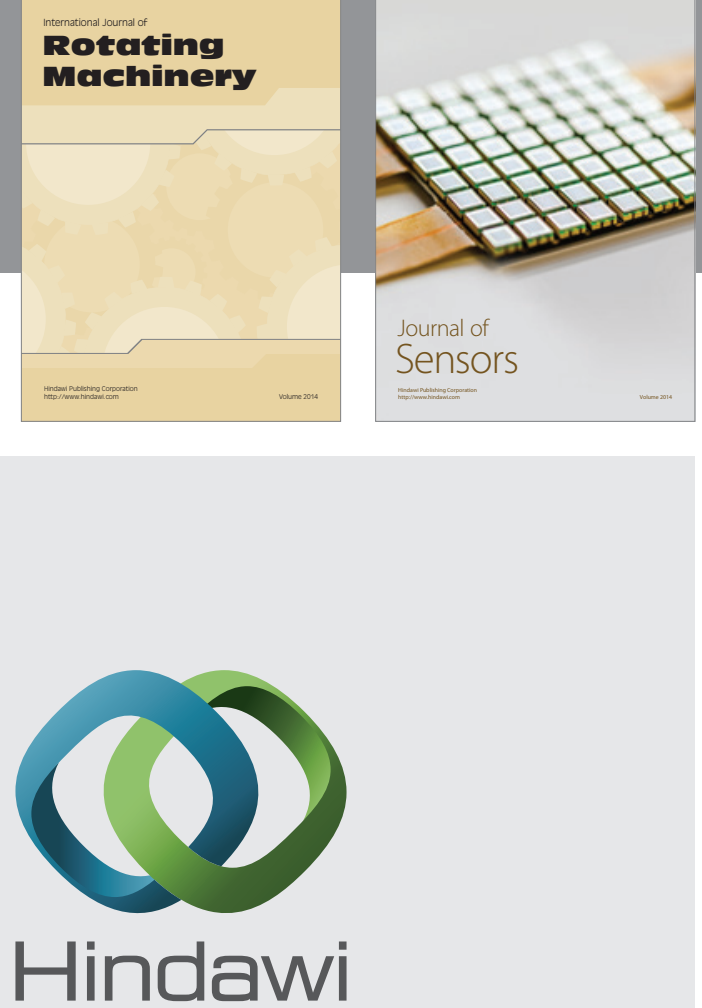

Submit your manuscripts at http://www.hindawi.com
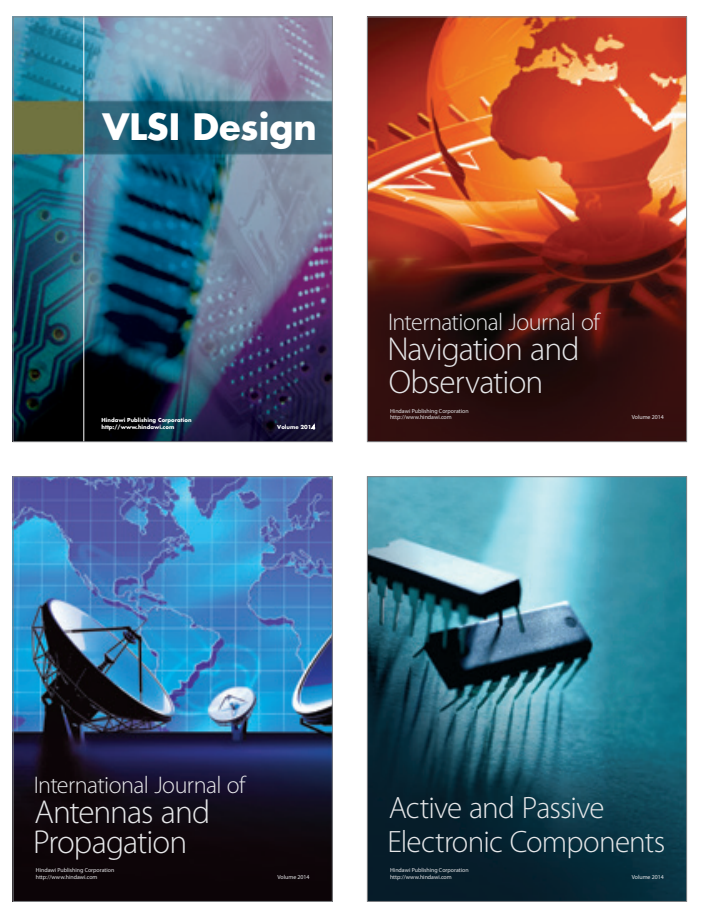
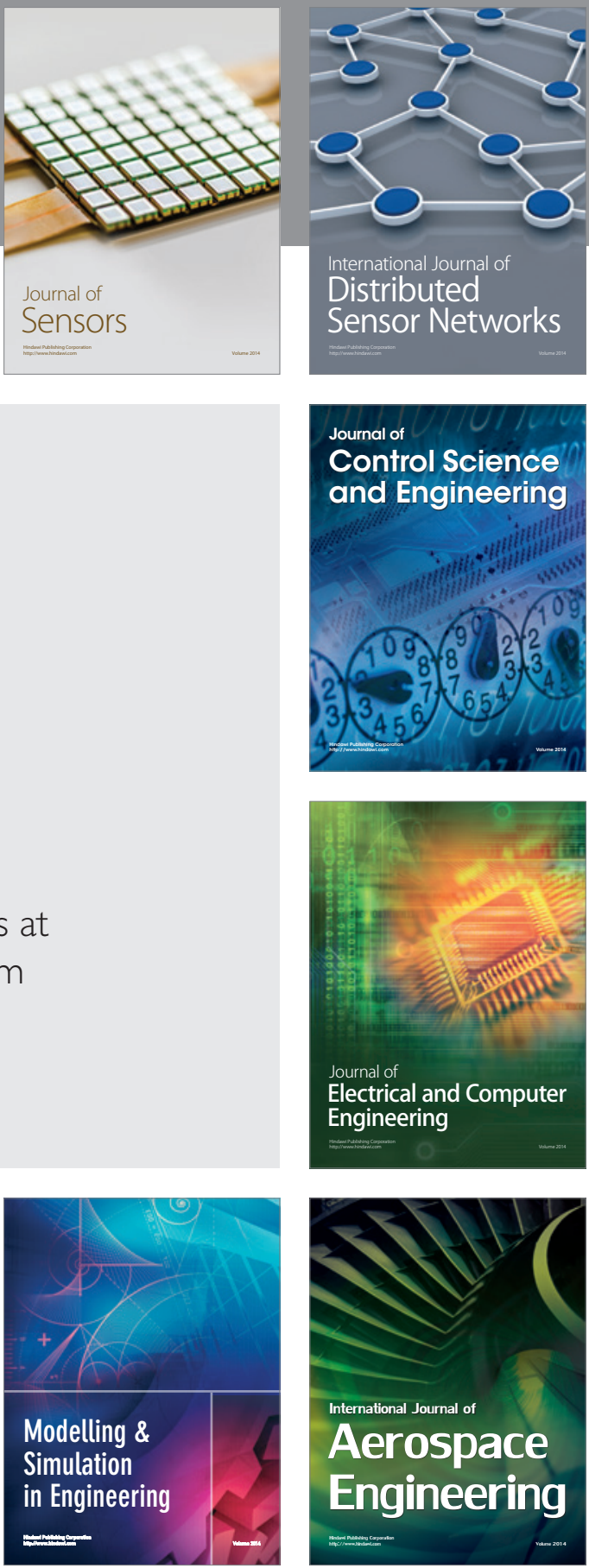

Journal of

Control Science

and Engineering
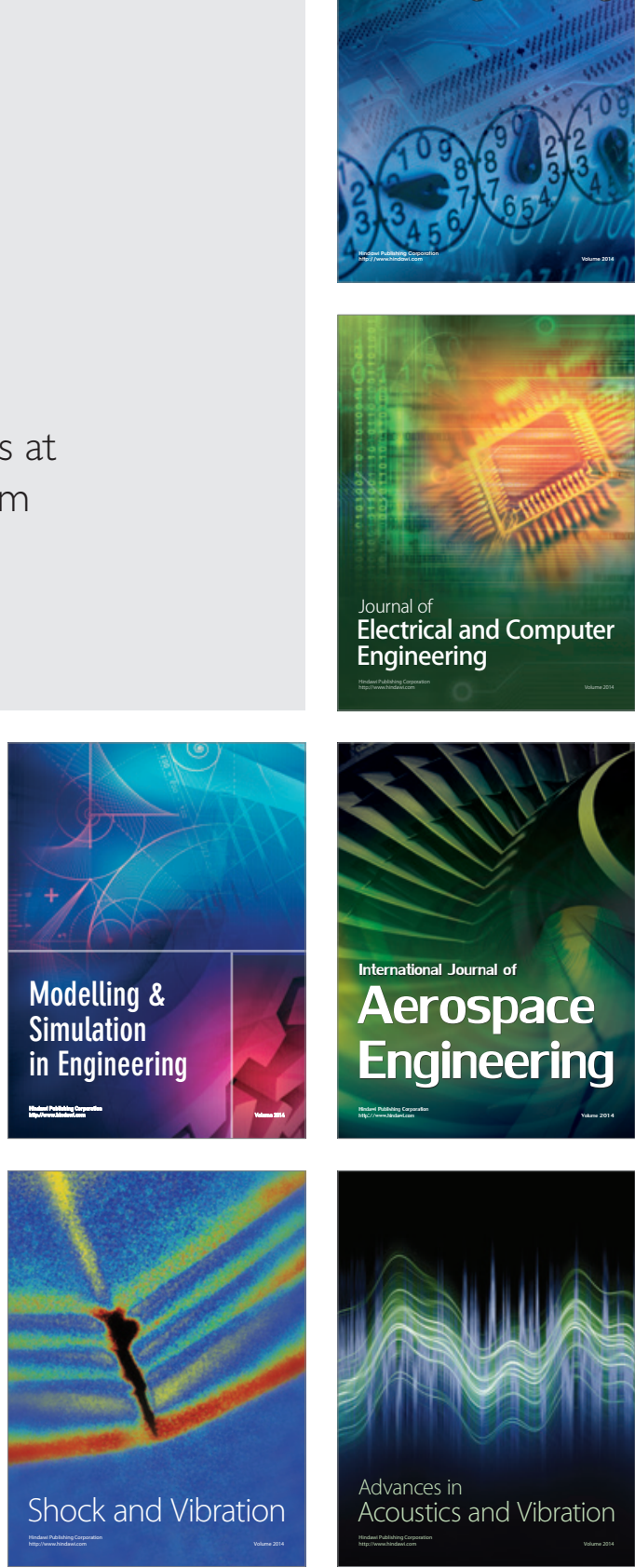